\title{
The Global Biosurveillance Portal: Biosurveillance for the Department of Defense
}

\author{
Janelle A. Anderson*, C. Nicole Rosenzweig, Jason Roos and Brandon Flores \\ Joint Program Executive Office for Chemical and Biological Defense, Aberdeen Proving Ground, MD, USA
}

\section{Objective}

This presentation aims to describe the development, initial use, and future directions of the Biosurveillance Portal.

\section{Introduction}

The 2012 National Strategy for Biosurveillance ${ }^{1}$ calls for improved integration, synchronization, and coordination of national biosurveillance activities and acknowledges the benefits of collective knowledge through sharing and receiving of biosurveillance information via strengthened partnerships amongst international, federal, state, local, tribal, territorial, private sector, nongovernmental, academic and other participants. To assist in meeting these goals and goals specifically called out by United States (U.S.) Military and international partners, the Joint Program Executive Office for Chemical and Biological Defense began developing a software suite to support biosurveillance needs. In October 2014, the Biosurveillance Portal (BSP) will be deployed to support biosurveillance operations in the Korean Theater. In October 2016, the BSP will be available globally.

\section{Methods}

System Development

The BSP is being developed on the Ozone Widget Framework (OWF) architecture, which will allow users to organize and use multiple web applications in a single window. The BSP will be a system of systems and will pull in capabilities and data streams that are already available across the Department of Defense (DoD), other government agencies and industry. For example, there is a plan in place for data and information to be ingested from the Armed Forces Health Surveillance Center, the U.S. Army Public Health Command, and the Centers for Disease Control and Prevention, as well as from feeds from the World Health Organization, and the Program for Monitoring Emerging Diseases. Many other government systems are also being developed on the OWF, which allows all of these systems to easily integrate, as needed, and allows the U.S. government to produce multiple types of systems in the most cost efficient manner without duplicating efforts.

BSP developers are using an agile development approach with a cycle of one month sprints, and three month capability drops. Each capability drop delivery is followed up with a user feedback event where users test and evaluate the capabilities that have been delivered.

Requirements

The U.S. Special Operations Command (USSOCOM) recognized that no current capability exists to support the National Strategy's intent. In addition, USSOCOM requires a biosurveillance system to close information gaps that presently delay decision making at the tactical, operational and strategic levels, and inhibit effective planning and response. The Korean BSP was recognized by USSOCOM as the potential tool that following modifications could be used to meet their biosurveillance needs.

USSOCOM biosurveillance data and information requirements as well as system requirements were laid out in a requirements document that was validated in May 2014. For each data requirement potential data providers have been identified from offices within the DoD, other federal government agencies, and private organizations. BSP developers will work with these data providers to determine how their data will be ingested by the BSP with data agreements put in place as needed.

It is anticipated that other U.S. Combatant Commands (CCMDs) have biosurveillance needs that are similar to those of USSOCOM. Because of this, BSP developers are also engaging key stakeholders at other CCMDs in order to document and incorporate their requirements into the BSP.

\section{Results}

The BSP was used as the primary communication tool during a trilateral whole of government biological event exercise involving the U.S., Republic of Korea, and Australia. The first operational release will represent the first fully integrated DoD network accredited capability set and is planned for deployment in October 2014.

\section{Conclusions}

The BSP has already proven itself as an effective communication tool that can be used during biological event exercises. As the BSP matures it will provide information sharing across the DoD biosurveillance community of interest, other U.S. Government agencies, and foreign government agencies to promote a "whole of government" biosurveillance capability. Ultimately, the BSP will facilitate collaboration, communication, information sharing, and provide a centralization of biosurveillance resources in support of the detection, management and mitigation of man-made and naturally occurring events.

\section{Keywords}

biosurveillance; Department of Defense; communication

\section{References}

1. White House. (2012). National Strategy for Biosurveillance. Retrieved from http://www.whitehouse.gov/sites/default/files/National_ Strategy_for_Biosurveillance_July_2012.pdf

\footnotetext{
* Janelle A. Anderson

E-mail: janelle.a.anderson.civ@mail.mil
} 